\title{
Nghiên cứu các yếu tố ảnh hưởng đến mức độ khó khăn vươong mắc trong công tác quản lý chi phí đầu tư xây dựng
}

\author{
Lê Trung Phong ${ }^{1^{*}}$, Mai Bá Nhẫn ${ }^{1}$
}

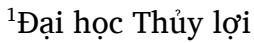

\begin{tabular}{|c|c|}
\hline TỪ KHOÁ & TÓM TẮT \\
\hline Quản lý chi phí & Bài báo trình bày kết quả tìm ra các yếu tố ảnh hưởng đến mức độ khó khăn vướng mắc trong công tác \\
\hline Chi phí đầu tư xây dựng & quản lý chi phí đầu tư xây dựng, từ đó đề xuất các giải pháp phù hợp. Tác giả đã thực hiện nghiên cứu \\
\hline Nhân tố ảnh hưởng & 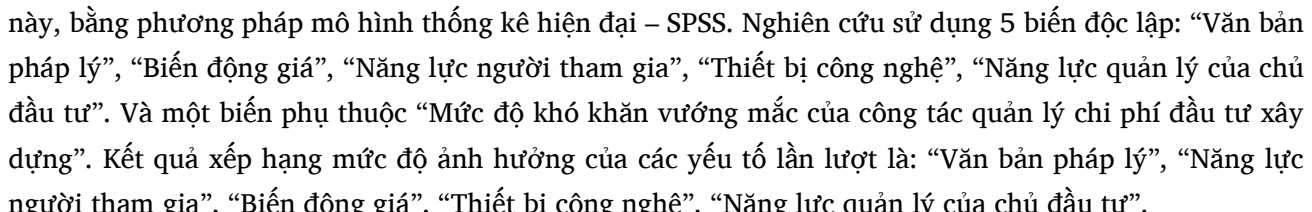 \\
\hline
\end{tabular}

\section{KEYWORDS}

\section{Cost management}

Construction investment costs Influential factors

\section{ABSTRACT}

To find out the factors that affect the difficulty level in management of construction investment costs, from there propose suitable solutions. The author has done this study, by method - SPSS. There are 5 independent variables: "Law", "Price volatility", "Personnel”, "Technology equipment", "Investor". There is 1 dependent variable: "Difficulty level of construction investment cost management". Ranking results: "Law", "Personnel”, "Price volatility”, “Technology equipment”, "Investor".

\section{Mở đầu}

Quản lý chi phí đầu tư xây dựng có tầm quan trọng to lớn, nó diễn ra trong suốt quá trình hình thành, triển khai thực hiện đến kết thúc dự án. Quyết định đến hiệu quả sử dụng đồng vốn của Dự án.

Tuy nhiên, trong thời gian qua chứng kiến hàng loạt các dự án rơi vào tình trạng vượt giá trị ước tính ban đầu, chậm tiến độ giải ngân vốn. Cho thấy công tác quản lý chi phí đầu tư xây dựng hiện nay, đang gặp nhiều khó khăn vướng mắc .

Với mong muốn, tìm ra các yếu tố ảnh hưởng và mức độ ảnh hưởng của từng yếu tố. Từ đó đưa ra các đề xuất giải pháp phù hợp . Tác giả lựa chọn nghiên cứu đề tài: "Nghiên cứu các yếu tố ảnh hưởng đến mức độ khó khăn vướng mắc trong công tác quản lý chi phi phí đầu tư xây dựng".

Bằng phương pháp mô hình thống kê hiện đại - SPSS, tác giả tiến hành điều tra khảo sát, lấy ý kiến các chuyên gia, nhà quản lý và những người đang tham gia trong lĩnh vực xây dựng. Sau đó phân tích, đánh giá và đề xuất giải pháp nhằm khắc phục các khó khăn vướng mắc trên.

\section{Phương pháp nghiên cứu}

Thông qua các kết quả nghiên cứu liên quan, đã được thực hiện trong và ngoài nước, tác giả bước đầu nhận dạng được một số yếu tố ảnh hưởng đến mức độ khó khăn vướng mắc trong công tác quản lý chi phí đầu tư xây dựng. Sau đó, tiến hành tham khảo ý kiến trực tiếp từ một số chuyên gia quen biết, bằng hình thức trò chuyện.

Tác giả lọc ra được 5 yếu tố có khả năng ảnh hưởng đến mức độ khó khăn vướng mắc trong công tác quản lý chi phí đầu tư xây dựng. Với các giả thuyết được đặt ra như sau:

- H1: Ảnh hưởng của các yếu tố văn bản pháp lý càng lớn, thì mức độ khó khăn vướng mắc của công tác quản lý chi phí đầu tư xây dựng càng nghiêm trọng

- H2: Ảnh hưởng của các yếu tố biến động giá thị trường càng lớn, thì mức độ khó khăn vướng mắc của công tác quản lý chi phí đầu tư xây dựng càng nghiêm trọng

- H3: Ảnh hưởng của các yếu tố từ năng lực, kinh nghiệm và ý thức trách nhiệm người tham gia càng lớn, thì mức độ khó khăn vướng mắc của công tác quản lý chi phí đầu tư xây dựng càng nghiêm trọng

- H4: Ảnh hưởng của các yếu tố từ thiết bị công nghệ càng lớn, thì mức độ khó khăn vướng mắc của công tác quản lý chi phí đầu tư xây dựng càng nghiêm trọng

- H5: Ảnh hưởng của các yếu tố về năng lực quản lý công tác lập, thẩm tra, phê duyệt thiết kế dự toán của Chủ đầu tư càng lớn, thì mức độ 
khó khăn vướng mắc của công tác quản lý chi phí đầu tư xây dựng càng nghiêm trọng.

Ta có mô hình nghiên cứu như sau:

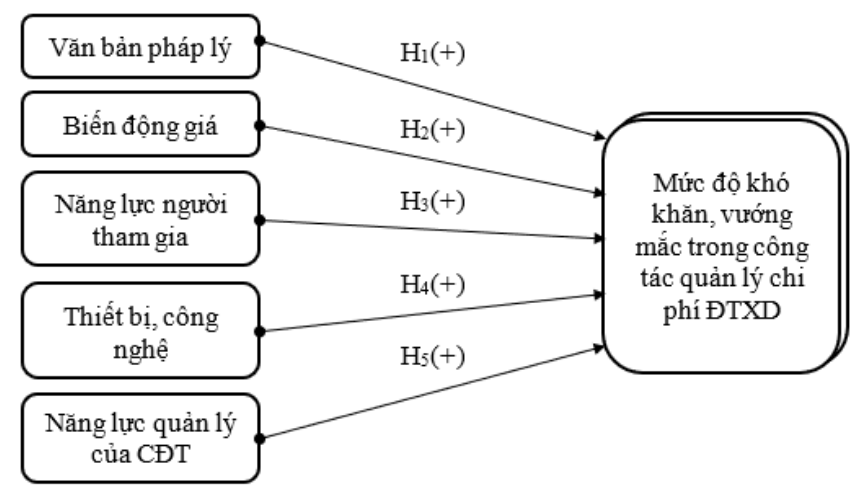

Hình 1. Mô hình nghiên cứu được lựa chọn.

Trong đó biến độc lập là: Văn bản pháp lý, Biến động giá, Năng lực của người tham gia, Thiết bị công nghệ, Năng lực quản lý của chủ đầu tư.

Biến phụ thuộc là: Mức độ khó khăn vướng mắc trong công tác quản lý chi phí đầu tư xây dựng.

Mẫu được chọn theo phương pháp lấy mẫu thuận tiện. Theo Hoàng Trọng, Chu Nguyễn Mộng Ngọc (2008) [1] cho rằng cỡ mẫu ít nhất phải bằng 4 hoặc 5 lần số biến quan sát trong phân tích nhân tố.

\subsection{Phương pháp thu thập số liệu}

Bảng câu hỏi khảo sát được xây dựng theo thang đo Likert 5 mức độ. Nội dung bảng khảo sát được chia làm 2 phần:

Phần A: Thông tin chung, bao gồm các thông tin chung về nơi công tác, thâm niên, chức vụ, loại dự án đã tham gia,... của các cá nhân tham gia khảo sát.

Phần B: Đánh giá mức độ đồng ý về các thực trạng của các yếu tố liên quan đến sự khó khăn vướng mắc trong công tác quản lý chi phí đầu tư xây dựng. Với 5 mức độ:

Bảng 1.

Thang đo Likert 5 mức độ.

\begin{tabular}{|c|c|c|c|c|}
\hline 1 & 2 & 3 & 4 & 5 \\
\hline $\begin{array}{c}\text { Hoàn toàn } \\
\text { không đồng } \\
\text { ý }\end{array}$ & $\begin{array}{c}\text { Không } \\
\text { đồng ý } \\
\text { vừa }\end{array}$ & $\begin{array}{c}\text { Không ý } \\
\text { kiến }\end{array}$ & $\begin{array}{c}\text { Đồng ý } \\
\text { vừa }\end{array}$ & $\begin{array}{c}\text { Hoàn toàn } \\
\text { đồng ý }\end{array}$ \\
\hline
\end{tabular}

\subsection{Phương pháp phân tích}

Kiểm định độ tin cậy của thang đo Cronbach's Alpha
Nhằm kiểm tra các biến quan sát của thang đo, có thể hiện được đặc điểm và tính chất của nhân tố mẹ hay không. Nếu một thang đo mà có độ tin cậy thấp thì sẽ bị loại bỏ. Việc này có ý nghĩa rất lớn đến mức độ tin cậy của các câu hỏi và kết quả phân tích. Một thang đo được đánh giá tốt khi có hệ số Cronbach's Alpha lớn hơn hoặc bằng 0,7 theo Nunnally \& Bernstein, 1994 được trích bởi Nguyễn Đình Thọ (2013) [2]. Ngoài ra, biến quan sát phải có hệ số tương quan biến tổng phải lớn hơn hoặc bằng 0,3 mới đạt yêu cầu.

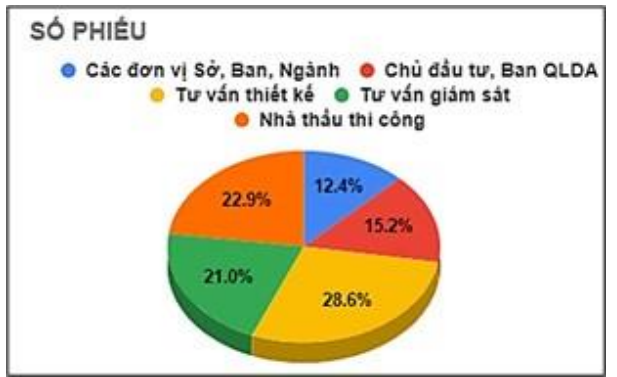

Hình 2. Thống kê nơi công tác.

Phân tích nhân tố khám phá (EFA)

Nhằm rút gọn một tập $\mathrm{k}$ biến quan sát thành một tập $\mathrm{F}$ (với $\mathrm{F}<\mathrm{k}$ ) các nhân tố có ý nghĩa hơn. Và xem xét mối quan hệ giữa các biến ở tất cả các nhân tố khác nhau, nhằm phát hiện ra những biến quan sát trùng lắp ở nhiều nhân tố hoặc các biến quan sát bị phân sai nhân tố từ ban đầu.

Phương pháp phân tích nhân tố khám phá được sử dụng khi hệ số KMO có giá trị từ 0,5 trở lên. Mức ý nghĩa thống kê sig Bartlett's Test phải nhỏ hơn 0,05 theo Hoàng Trọng, Chu Nguyễn Mộng Ngọc (2008) [1] Thang đo được chấp nhận khi tổng phương sai trích (Total Variance Explained) lớn hơn hoặc bằng $50 \%$. Trị số Eigenvalue lớn hơn hoặc bằng 1 . Kích thước mẫu là 104 nên hệ số tải nhân tố (Factor loading) $\geq$ 0,55 theo Nguyễn Đình Thọ (2013) [2].

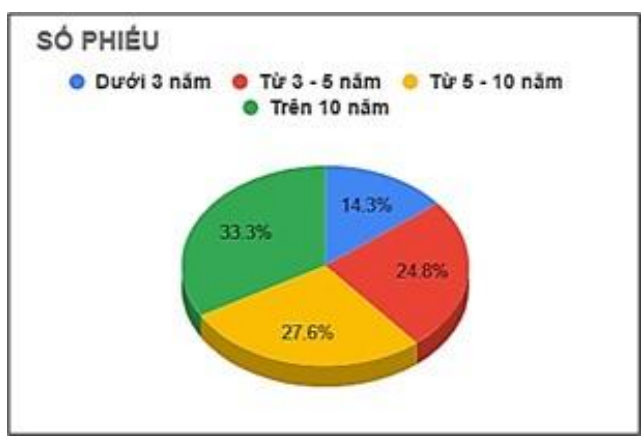

Hình 3. Thống kê thâm niên. 


\section{Phân tích hồi quy tuyến tính bội}

Nhằm xác định vai trò quan trọng của từng nhân tố tác động đến mức độ khó khăn vướng mắc trong công tác quản lý chi phí đầu tư xây dựng.

Sử dụng phương pháp Enter trong SPSS. Hệ số $\mathrm{R}^{2}$ điều chỉnh phản ánh mức độ ảnh hưởng của các biến độc lập lên biến phụ thuộc. Thường thì giá trị này từ $50 \%$ trở lên là nghiên cứu được đánh giá tốt. Hệ số Durbin-Watson (DW) dùng để kiểm định sự tương quan chuỗi bậc nhất giữa các sai số liền kề nhau $(1<\mathrm{DW}<3)$. Kiểm tra hiện tượng đa cộng tuyến bằng hệ số phóng đại phương sai VIF < 10 [1].

Mô hình hồi quy tuyến tính bội có dạng như sau:

$\mathrm{Y}=\beta 0+\beta 1 \mathrm{X} 1+\beta 2 \mathrm{X} 2+\beta 3 \mathrm{X} 3+\beta 4 \mathrm{X} 4+\beta 5 \mathrm{X} 5+\varepsilon(1)$

$\mathrm{Y}=$ Mức độ khó khăn vướng mắc

X1: Yếu tố văn bản pháp lý; X2: Yếu tố biến động giá;

X3: Yếu tố năng lực người tham gia; X4: Yếu tố thiết bị công nghệ;

X5: Yếu tố năng lực quản lý của chủ đầu tư;

$\beta 0$ : Hệ số góc; $\beta 1 \div \beta 5$ : Các hệ số hồi quy riêng; ع: Sai số

\section{Phân tích phương sai một yếu tố (One-Way ANOVA)}

Phân tích ANOVA nhằm phân tích có hay không sự khác biệt về đánh giá mức độ khó khăn vướng mắc trong công tác quản lý chi phí đầu tư xây dựng từ các giới tính, chức vụ, nơi công tác, thâm niên khác nhau.

\section{Thu thập và phân tích số liệu}

\subsection{Khảo sát thử nghiệm}

Ở giai đoạn 1 , tác giả tiến hành khảo sát thử nghiệm, thực hiện thông qua phương pháp thảo luận lấy ý kiến trực tiếp từ một số chuyên gia quen biết, bằng hình thức trao đổi trò chuyện và gửi email. Nhằm kiểm tra sơ lược các yếu tố ảnh hưởng và hoàn thiện bảng câu hỏi khảo sát, phục vụ cho công tác điều tra khảo sát chính thức ở giai đoạn 2.

\subsection{Khảo sát chính thức}

Nghiên cứu này có tất cả 21 biến quan sát cần tiến hành phân tích nhân tố, Vì vậy kích thước mẫu yêu cầu tối thiểu là 21 x $5=105$ quan sát. Tác giả thực hiện khảo sát từ tháng 11/2020 đến tháng 01/2021. Có 110 phiếu khảo sát được phát đi và thu về 105 mẫu hợp lệ, trong đó các mẫu bị loại là do điền không đủ thông tin.

Hình thức khảo sát được thực hiện trực tiếp tại các công ty xây dựng, phòng quản lý dự án, công trường xây dựng và một số nơi khác.

Thông qua Hình 2, ta thấy toàn bộ các cá nhân tham gia khảo sát đều đang làm việc liên quan đến xây dựng. Tại nhiều tổ chức khác nhau, nhằm đa dạng hóa đối tượng khảo sát và phản ánh tương đối sát thực tế.

Thâm niên của người tham gia phỏng vấn đóng vai trò rất quan trọng đến chất lượng của kết quả khảo sát. Hiểu biết và kinh nghiệm của họ càng nhiều thì sự nhìn nhận đánh giá sẽ càng chính xác và khách quan hơn.

Thông qua Hình 3 , ta thấy các thành phần tham gia khảo sát có thâm niên từ 5 năm trở lên chiếm đa số (60,9\%), thâm niên dưới 5 năm là (39,1 \%).

Thành phần tham gia khảo sát theo Hình 4 là khá đa dạng và đầy đủ các chức vụ, thường xuyên tiếp xúc và liên quan đến công tác quản lý chi phí đầu tư xây dựng. Việc này sẽ giúp kết quả khảo sát có ý nghĩa tổng quát và khách quan hơn.

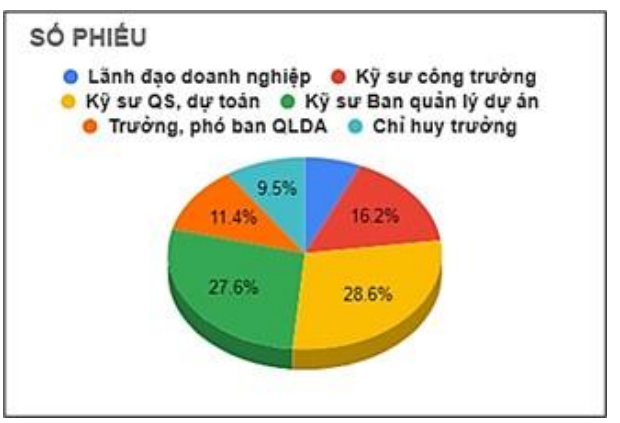

Hình 4. Thống kê chức vụ.

Từ biểu đồ Hình 5, thực trạng về mức độ bất cập, khó khăn, vướng mắc trong công tác quản lý chi phí đầu tư xây dựng hiện nay là cao và rất cao (chiếm 74,3 \%), rất ít và trung bình (chiếm 25,7 \%). Chứng tỏ đề tài nghiên cứu có tính thực tiễn cao.

Từ biểu đồ Hình 6 , tỷ lệ cần thiết thực hiện nghiên cứu này là $55,2 \%$, rất cần thiết là $44,8 \%$, không cần thiết là $0 \%$, chứng tỏ việc nghiên cứu đề tài này có yêu cầu cấp thiết cao.

\section{Kết quả nghiên cứu}

4.1. Kiểm định độ tin cậy thang đo Cronbach's Alpha

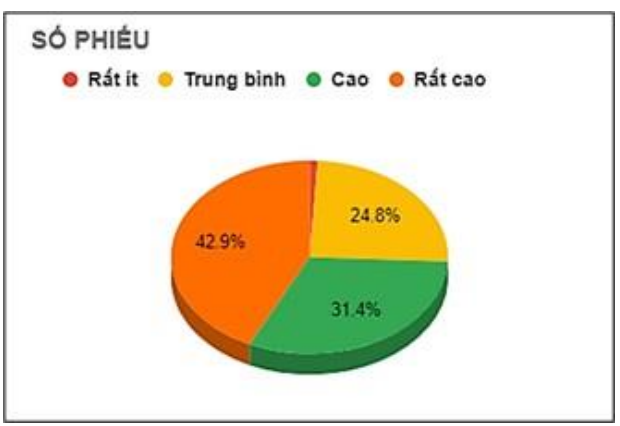

Hình 5. Thống kê mức độ khó khăn vướng mắc trong công tác quản lý chi phí đầu tư xây dựng. 
Kiểm định lần 1 đã loại bỏ 3 biến rác có hệ số tương quan với biến tổng không đạt điều kiện $(<0,3)$, bao gồm biến con PL5 của yếu tố Văn bản pháp luật, GTT5 của yếu tố Biến động giá, NS5 của yếu tố Năng lực của người tham gia.

Sau đó kiểm định lần 2, kết quả hệ số Cronbach's Alpha cho ra $>0,7$ và hệ số tương quan biến tổng nhỏ nhất $>0,3$. Ta loại 3 biến rác trên, tiến hành phân tích nhân tố khám phá với 18 biến còn lại.

\subsection{Phân tích nhân tố khám phá (EFA)}

Bảng 2.

Bảng phân tích hệ số KMO và kiểm định Bartlett.

\begin{tabular}{|l|l|l|}
\hline \multicolumn{3}{|c|}{ Bài kiểm tra KMO và Bartlett } \\
\hline \multicolumn{2}{|c|}{ Thang đo độ đầy đủ Kaiser-Meyer-Olkin } & 0,796 \\
\hline \multirow{2}{*}{$\begin{array}{l}\text { Bài kiểm tra độ cầu } \\
\text { thể Barlett }\end{array}$} & Số chi bình phương xấp xỉ & 1283,707 \\
\cline { 2 - 3 } & $\mathrm{df}$ & 153 \\
\cline { 2 - 3 } & Sig. & 0,000 \\
\hline
\end{tabular}

\section{Bảng 3.}

Bảng phân tích thành phần nguyên gốc sau khi phân tích nhân tố.

\begin{tabular}{|l|l|l|}
\hline \multicolumn{3}{|c|}{ Độ tương quan } \\
\hline & Khởi điềm & Được tách \\
\hline PL1 & 1,000 & 0,884 \\
\hline PL2 & 1,000 & 0,823 \\
\hline PL3 & 1,000 & 0,886 \\
\hline PL4 & 1,000 & 0,887 \\
\hline GTT1 & 1,000 & 0,834 \\
\hline GTT2 & 1,000 & 0,862 \\
\hline GTT3 & 1,000 & 0,866 \\
\hline GTT4 & 1,000 & 0,871 \\
\hline NS1 & 1,000 & 0,679 \\
\hline NS2 & 1,000 & 0,640 \\
\hline NS3 & 1,000 & 0,721 \\
\hline NS4 & 1,000 & 0,647 \\
\hline CN1 & 1,000 & 0,711 \\
\hline CN2 & 1,000 & 0,710 \\
\hline CN3 & 1,000 & 0,781 \\
\hline CDT1 & 1,000 & 0,791 \\
\hline CDT2 & 1,000 & 0,882 \\
\hline CDT3 & 1,000 & 0,844 \\
\hline Phương pháp tách: Phân tích cấu kiện nguyên chất \\
\hline
\end{tabular}

Theo Bảng 2, ta có hệ số KMO = 0,796 > 0,5, với mức ý nghĩa Sig $=0,000>0,05$ chứng tỏ mô hình đưa vào phân tích nhân tố là thích hợp.
Theo Bảng 3, hệ số Extraction nhỏ nhất của tất cả các biến quan sát là $0,640>0,5$ chứng tỏ 18 biến quan sát đều đạt yêu cầu và được giữa lại trong phân tích nhân tố khám phá.

\section{Bảng 4.}

Ma trận xoay 5 yếu tố tác động đến công tác quản lý chi phí đầu tư xây dựng.

\begin{tabular}{|l|l|l|l|l|l|}
\hline \multicolumn{7}{|l|}{ Ma trận cấu kiện đã xoay } \\
\hline & \multicolumn{1}{|l|}{ Cấu kiện } & 2 & 3 & 4 & 5 \\
\hline & 1 & & & & \\
\hline GTT4 & 0,928 & & & & \\
\hline GTT3 & 0,924 & & & & \\
\hline GTT2 & 0,923 & & & & \\
\hline GTT1 & 0,903 & & & & \\
\hline PL3 & & 0,937 & & & \\
\hline PL4 & & 0,934 & & & \\
\hline PL1 & & 0,931 & & & \\
\hline PL2 & & 0,890 & & & \\
\hline NS3 & & & 0,835 & & \\
\hline NS4 & & & 0,794 & & \\
\hline NS2 & & & 0,791 & & \\
\hline NS1 & & & 0,784 & & 0,797 \\
\hline CDT2 & & & & 0,935 & \\
\hline CDT3 & & & & 0,908 & \\
\hline CDT1 & & & & 0,834 & \\
\hline CN3 & & & & & 0,874 \\
\hline CN2 & & & & & 0,810 \\
\hline CN1 & & & & & \\
\hline
\end{tabular}

Theo Bảng 5 , giá trị trích Eigenvalue $=1,540>1$ điều này có nghĩa là chỉ những nhân tố được trích ra có Eigenvalue lớn hơn 1 mới được giữ lại trong mô hình phân tích nhân tố. Với 18 biến quan sát được đưa vào phân tích theo tiêu chuẩn Eigenvalue lớn hơn 1, có 5 nhân tố được rút trích. Tổng phương sai trích được từ 5 nhân tố này là 79,485 \%, có nghĩa là 5 nhân tố này giải thích được 79,485 \% biến thiên của dữ liệu. Như vậy, thang đo các nhân tố trích ra đều đạt được độ tin cậy và giá trị.

Theo Bảng 4, ta có mô hình nghiên cứu điều chỉnh như sau:

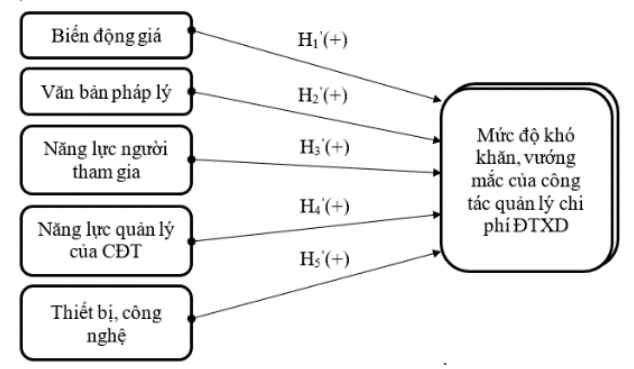

Hình 6. Mô hình nghiên cứu điều chỉnh 


\section{Bảng 5.}

Bảng tổng phương sai trích.

\begin{tabular}{|c|c|c|c|c|c|c|c|c|c|}
\hline \multicolumn{10}{|c|}{ Giải thích tổng phương sai } \\
\hline Cấu & \multicolumn{3}{|c|}{ Giá trị riêng khởi điểm } & \multicolumn{3}{|c|}{ Tổng tách ra từ lượng tải bình phương } & \multicolumn{3}{|c|}{ Tổng xoay từ lượng tải bình phương } \\
\hline & Tổng & \% phương sai & $\%$ tích lũy & Tổng & $\%$ phương sai & $\%$ tích lũy & Tồng & \% phương sai & $\%$ tích lũy \\
\hline 1 & 4,397 & 24,426 & 24,426 & 4,397 & 24,426 & 24,426 & 3,492 & 19,400 & 19,400 \\
\hline 2 & 3,396 & 18,865 & 43,291 & 3,396 & 18,865 & 43,291 & 3,482 & 19,347 & 38,747 \\
\hline 3 & 2,637 & 14,652 & 57,944 & 2,637 & 14,652 & 54,944 & 2,625 & 14,583 & 53,330 \\
\hline 4 & 2,338 & 12,989 & 70,932 & 2,338 & 12,989 & 70,932 & 2,501 & 13,893 & 67,223 \\
\hline 5 & 1,540 & 8,553 & 79,485 & 1,540 & 8,553 & 79,485 & 2,207 & 12,226 & 79,485 \\
\hline 6 & 0,599 & 3,329 & 82,814 & & & & & & \\
\hline 7 & 0,520 & 2,889 & 85,703 & & & & & & \\
\hline 8 & 0,408 & 2,268 & 87,971 & & & & & & \\
\hline 9 & 0,384 & 2,132 & 90,104 & & & & & & \\
\hline 10 & 0,356 & 1,977 & 92,081 & & & & & & \\
\hline 11 & 0,260 & 1,447 & 93,528 & & & & & & \\
\hline 12 & 0,238 & 1,320 & 94,848 & & & & & & \\
\hline 13 & 0,202 & 1,121 & 95,969 & & & & & & \\
\hline 14 & 0,186 & 1,032 & 97,001 & & & & & & \\
\hline 15 & 0,152 & 0,844 & 97,845 & & & & & & \\
\hline 16 & 0,148 & 0,820 & 98,665 & & & & & & \\
\hline 17 & 0,133 & 0,737 & 99,402 & & & & & & \\
\hline 18 & 0,108 & 0,598 & 100,000 & & & & & & \\
\hline
\end{tabular}

\subsection{Phân tích hồi quy đa biến}

Kết quả phân tích hồi quy Bảng 6 , có $\mathrm{R} 2=0,528$ và hệ số $\mathrm{R} 2$ điều chỉnh là 0,504 . Điều đó cho thấy độ thích hợp của mô hình là 50,4 \% hay nói cách khác là 50,4 \% độ biến thiên về yếu tố gây khó khăn vướng mắc cho công tác quản lý chi phí đầu tư xây dựng được giải thích chung bởi các biến độc lập trong mô hình. Còn lại là do sự tác động của các yếu tố khác chưa được đưa vào mô hình.

\section{Bảng 6.}

Các tham số thống kê từ phân tích hồi quy đa biến.

\begin{tabular}{|l|l|l|l|l|l|}
\hline \multicolumn{5}{|c|}{ Tổng kết mô hình } \\
\hline Model & R & $\begin{array}{l}\text { R bình } \\
\text { phương }\end{array}$ & $\begin{array}{l}\text { R bình } \\
\text { phương đã } \\
\text { chỉnh }\end{array}$ & $\begin{array}{l}\text { Sai số chuẩn } \\
\text { của ước } \\
\text { lượng }\end{array}$ & $\begin{array}{l}\text { Chỉ số } \\
\text { Durbin- } \\
\text { Watson }\end{array}$ \\
\hline 1 & $0,726^{\text {a }}$ & 0,528 & 0,504 & 0,587 & 1,724 \\
\hline
\end{tabular}

a. Predictors: (Constant), TB QL chủ đầu tư, TB nhân sự, TB văn bản PL, TB Biến động giá, TB công nghệ

b. Biến phụ thuộc: mức độ khó khăn vướng mắc của công tác QLCP hiện nay
Bảng 7.

Thống kê phân tích phương sai ANOVA.

\begin{tabular}{|l|l|l|l|l|l|l|}
\hline \multicolumn{7}{|c|}{ ANOVA $^{\text {a }}$} \\
\hline $\begin{array}{l}\text { Mònh } \\
\text { hìn }\end{array}$ & $\begin{array}{l}\text { Tồng các } \\
\text { bình } \\
\text { phương }\end{array}$ & df & $\begin{array}{l}\text { Trung bình } \\
\text { các bình } \\
\text { phương }\end{array}$ & F & Sig. \\
\hline 1 & $\begin{array}{l}\text { Hồi } \\
\text { quy }\end{array}$ & 38,118 & 5 & 7,624 & 22,114 & $0,000^{\text {b }}$ \\
\hline & Số dư & 34,129 & 99 & 0,345 & & \\
\hline & & 72,248 & 104 & & & \\
\hline
\end{tabular}

a. Dependent Variable: Mức độ khó khăn vướng mắc của công tác QLCP hiện nay

b. Predictor: (Constant), TB QL chủ đầu tư, TB nhân sự, TB văn bản PL, TB biến động giá, TB công nghệ

Kết quả phân tích phương sai ANOVA Bảng 7 cho thây, giá trị thống kê $\mathrm{F}$ được tính từ giá trị R2 của mô hình có giá trị Sig. = 0,000. Điều này cho thấy, mô hình hồi quy tuyến tính bội phù hợp với tập dữ liệu, hay các biến độc lập có quan hệ với các biến phụ thuộc và mô hình có thể sử dụng được. 
Bảng 8.

Thống kê phân tích hệ số hồi quy Coefficients.

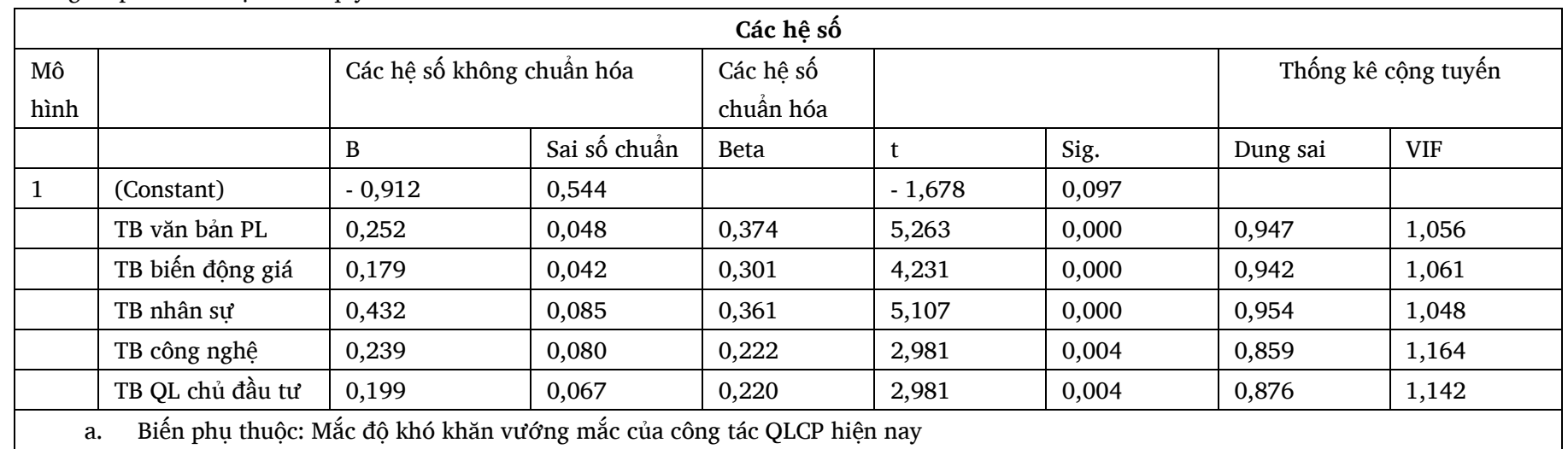

Kết quả phân tích hệ số hồi quy Coefficient Bảng 8 cho thây: Độ chấp nhận (Tolerance nhỏ nhất là 0,859 ) không quá thấp và hệ số phóng đại phương sai VIF (Variance Inflation Factor lớn nhất là 1,142) nhỏ hơn 10, (Hoàng Trọng, Chu Nguyễn Mộng Ngọc, 2008) [1], hệ số VIF vượt quá 10 thì đó là dấu hiệu của đa cộng tuyến. Do đó, các biến độc lập này không có quan hệ chặt chẽ với nhau nên không có hiện tượng đa cộng tuyến xảy ra. Vì vậy, tất cả 5 thành phần đo lường đều thỏa mãn yêu cầu. Tức là ta chấp nhận 5 giả thiết đã đặt ra, đó là giả thiết H1', H2', H3', H4' và H5'.

Ta có phương trình hồi quy bội như sau:

$\mathrm{Y}=-0,912+0,179 \mathrm{X} 1+0,52 \mathrm{X} 2+0,432 \mathrm{X} 3+0,199 \mathrm{X} 4+0,239 \mathrm{X} 5$ (1)

Trong đó: X1, X2, X3, X4, X5 là các yếu tố ảnh hưởng đến công tác quản lý chi phí (Y) tương ứng với các giả thuyết H'1, H'2, H’3, H'4 và H’5.

Theo Hoàng Trọng, Chu Nguyễn Mộng Ngọc (2008) [1], để đánh giá mức độ ảnh hưởng của các yếu tố, thì độ lớn của các hệ số phụ thuộc vào đơn vị đo lường của các biến, nên chỉ khi nào tất cả các biến độc lập đều có cùng đơn vị đo lường, thì các hệ số của chúng mới có thể so sánh trực tiếp với nhau. Một cách để làm cho các hệ số hồi quy có thể so sánh được với nhau là tính trọng số Beta, đó là hệ số của biến độc lập, khi tất cả dữ liệu trên các biến được biểu diễn bằng đơn vị đo lường độ lệch chuẩn.

Vì vậy, để so sánh xếp hạng mức độ ảnh hưởng của các yếu tố ta dùng hệ số Beta (hệ số chuẩn hóa - $\beta$ ) và phương trình hồi quy có thể viết lại như sau:

$$
\mathrm{Y}=0,301 \mathrm{X} 1+0,374 \mathrm{X} 2+0,361 \mathrm{X} 3+0,220 \mathrm{X} 4+0,222 \mathrm{X} 5
$$

\subsection{Dò tìm sự vi phạm giả đinh cần thiết trong hồi quy}

+ Giả định thứ nhất cần kiểm tra là giả định liên hệ tuyến tính. Phương pháp được sử dụng là biểu đồ phân tán Scatterplot với giá trị phần dư chuẩn hóa (Standarized residual) giá trị dự đoán chuẩn hóa (Standarized predicted value) trên trục hoành.

Từ đồ thị phân tán Scatterplot Hình 7 ta thấy, các phần dư phân tán ngẫu nhiên trong một vùng quanh đường đi qua tung độ 0 chứ không tạo thành một hình dạng nào. Điều này có ý nghĩa là giả thiết về sự tuyến tính không bị vi phạm.

+ Giả định thứ hai là giả định về phân phối chuẩn và phần dư. Sử dụng biểu đồ tần số (Histogram) của phần dư (đã được chuẩn hóa) để kiểm tra giả định này.

Kết quả từ biểu đồ tần số Histogram Hình 8 cho thấy, phân phối của phần dư xấp xỉ chuẩn (độ lệch chuẩn Std. Dev $=0,976$ gần bằng 1 ). Điều này có nghĩa là giả thiết phân phối chuẩn của phần dư không bị vi phạm.

+ Giả thiết thứ ba là giả thiết về tính độc lập của sai số (không có tương quan giữa các phần dư). Dùng đại lượng thống kê Durbin Watson (Bảng 6) để kiểm định. Theo trên trục tung và kết quả phân tích cho thấy giá trị $\mathrm{d}=1,724$, nằm trong khoảng từ 1 đến 3 , có nghĩa là $\mathrm{d}$ rơi vào miền chấp nhận giả thiết không có tương quan chuỗi bậc nhất với nhau. Do đó, giả thiết không có mối tương quan giữa các phần dư trong mô hình hồi quy đa biến không bị vi phạm.

+ Giả thiết thứ tư là giả thiết không có tương quan giữa các biến độc lập (đo lường đa cộng tuyến). Theo kết quả phân tích cho thấy hệ số phóng đại VIF thấp hơn 10 (Bảng 8). Do đó, giả thiết không có mối tương quan giữa các biến độc lập trong mô hình hồi quy không bị vi phạm.

+ Giả thiết thứ năm là các điểm phân vị trong phân phối của phần dư tập trung thành 1 đường chéo (Hình 9), như vậy giả định phân phối chuẩn của phần dư không bị vi phạm. 
Kết luận: Từ các kết quả kiểm tra trên cho thấy mô hình hồi quy xây dựng không vi phạm các giả thiết cần thiết trong hồi quy tuyến tính bội.

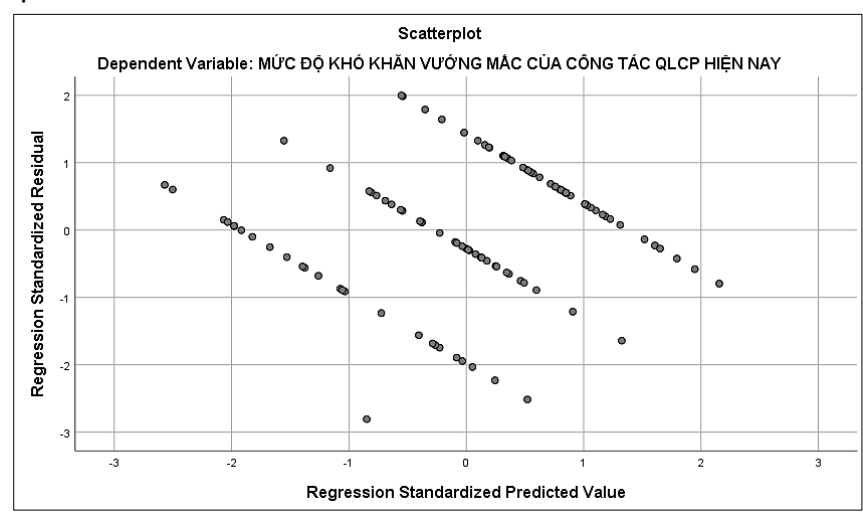

Hình 7. Đồ thị phân tán Scatterplot.

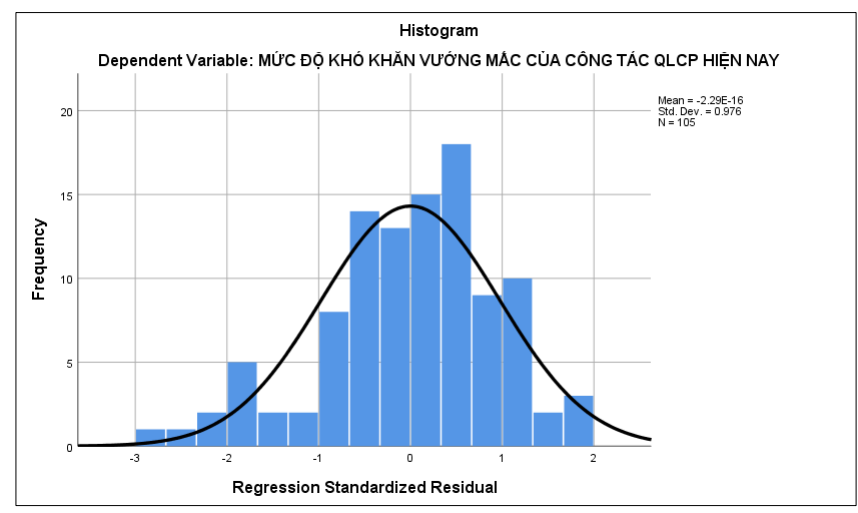

Hình 8. Đồ thị tần số Histogram.

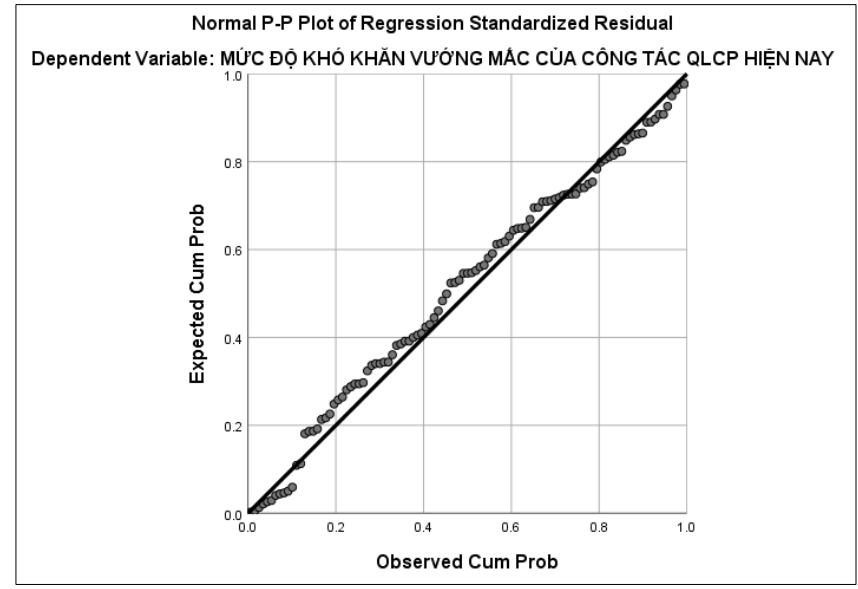

Hình 9. Biểu đồ phần dư chuẩn hóa Normal P-P Plot.

4.5. Phân tích phương sai một yếu tố (One_Way ANOVA)
Tiến hành kiểm định phương sai ANOVA với yếu tố giới tính, chức vụ, thâm niên trong nghề, vị trí công tác.

\section{Bảng 9.}

Kiểm tra tính đồng nhất của các phương sai đối với giới tính.

\begin{tabular}{|l|l|l|l|l|}
\hline \multicolumn{5}{|c|}{ Kiểm tra tính đồng nhất các phương sai } \\
\hline \multicolumn{4}{|c|}{ Mức độ khó khăn vướng mắc của công tác QLCP hiên nay } \\
\hline & Thống kê & df1 & Df2 & Sig. \\
\hline Welch & 0,101 & 1 & 15,642 & 0,754 \\
\hline \multicolumn{3}{|c|}{ a. Asymptotically F distributed } \\
\hline
\end{tabular}

Theo kết quả kiểm tra tính đồng nhất của các phương sai, với mức ý ngĩa Sig. = 0,754 > 0,05 (Bảng 9), có thể nói phương sai đánh giá mức độ khó khăn vướng mắc giữa Nam và Nữ không khác nhau một cách có ý nghĩa thống kê. Như vậy, kết quả phân tích ANOVA có thể sử dụng được.

\section{Bảng 10.}

Bảng kiểm tra kết quả phân tích ANOVA đối với mức độ khó khăn vướng mắc do cá nhân tham gia khảo sát.

\begin{tabular}{|c|c|c|c|c|c|}
\hline \multicolumn{6}{|c|}{ ANOVA } \\
\hline \multicolumn{6}{|c|}{ Mức độ khó khăn vướng mắc của công tác QLCP hiện nay } \\
\hline & $\begin{array}{c}\text { Tổng các } \\
\text { bình phương }\end{array}$ & $\mathrm{df}$ & $\begin{array}{c}\text { Trung bình các } \\
\text { bình phương }\end{array}$ & $\mathrm{F}$ & Sig. \\
\hline $\begin{array}{c}\text { GIữa các } \\
\text { nhóm }\end{array}$ & 0,070 & 1 & 0,070 & 0,100 & 0,752 \\
\hline $\begin{array}{c}\text { Trong } \\
\text { các } \\
\text { nhóm }\end{array}$ & 72,177 & 103 & 0,701 & & \\
\hline Tổng & 72,248 & 104 & & & \\
\hline
\end{tabular}

Theo kết quả phân tích ANOVA, với mức ý ngĩa Sig. = 0,752 > 0,05 (Bảng 10) nên có thể kết luận không có sự khác biệt có ý nghĩa thống kê về đánh giá mức độ khó khăn vướng mắc giữa các cá nhân tham gia khảo sát từ các giới khác nhau là nam và nữ.

\section{Bảng 11.}

Kiểm tra tính đồng nhất của các phương sai đối với chức vụ.

\begin{tabular}{|l|l|l|l|l|}
\hline \multicolumn{5}{|c|}{ Kiềm tra tính đồng nhất các phương sai } \\
\hline \multicolumn{5}{|c|}{ Mức độ khó khăn vướng mắc của công tác QLCP hiên nay } \\
\hline & Thống kê & df1 & Df2 & Sig. \\
\hline Welch & 1,990 & 5 & 30,045 & 0,109 \\
\hline \multicolumn{3}{|c|}{ a. Asymptotically F distributed } \\
\hline
\end{tabular}

Theo kết quả kiểm tra tính đồng nhất của các phương sai, với mức ý nghĩa Sig. $=0,109>0,05$ (Bảng 11), có thể nói phương sai 
đánh giá mức độ khó khăn vướng mắc giữa các chức vụ không khác nhau một cách có ý nghĩa thống kê. Như vậy, kết quả phân tích ANOVA có thể sử dụng được.

\section{Bảng 12.}

Bảng kiểm tra kết quả phân tích ANOVA đối với mức độ khó khăn vướng mắc do cá nhân tham gia khảo sát.

\begin{tabular}{|c|c|c|c|c|c|}
\hline \multicolumn{6}{|c|}{ ANOVA } \\
\hline \multicolumn{6}{|c|}{ Mức độ khó khăn vướng mắc của công tác QLCP hiện nay } \\
\hline & $\begin{array}{c}\text { Tổng các } \\
\text { bình phương }\end{array}$ & df & $\begin{array}{l}\text { Trung bình các } \\
\text { bình phương }\end{array}$ & $\mathrm{F}$ & Sig. \\
\hline $\begin{array}{c}\text { GIữa các } \\
\text { nhóm }\end{array}$ & 6,749 & 5 & 1,350 & 2,040 & 0,079 \\
\hline $\begin{array}{c}\text { Trong các } \\
\text { nhóm }\end{array}$ & 65,498 & 99 & 0,662 & & \\
\hline Tổng & 72,248 & 104 & & & \\
\hline
\end{tabular}

Theo kết quả phân tích ANOVA, với mức ý ngĩa Sig. = 0,079 > 0,05 (Bảng 12) nên có thể kết luận không có sự khác biệt có ý nghĩa thống kê về đánh giá mức độ khó khăn vướng mắc giữa các cá nhân tham gia khảo sát từ các chức vụ khác nhau.

\section{Bảng 13.}

Kiểm tra tính đồng nhất của các phương sai đối với thâm niên.

\begin{tabular}{|l|l|l|l|l|}
\hline \multicolumn{5}{|c|}{ Kiểm tra tính đồng nhất các phương sai } \\
\hline \multicolumn{5}{|c|}{ Mức độ khó khăn vướng mắc của công tác QLCP hiên nay } \\
\hline & Thống kê & df1 & Df2 & Sig. \\
\hline Welch & 2,102 & 3 & 45,838 & 0,113 \\
\hline \multicolumn{3}{|c|}{ a. Asymptotically F distributed } \\
\hline
\end{tabular}

\section{Bảng 14.}

Bảng kiểm tra kết quả phân tích ANOVA đối với mức độ khó khăn vướng mắc do cá nhân tham gia khảo sát.

\begin{tabular}{|c|c|c|c|c|c|}
\hline \multicolumn{6}{|c|}{ ANOVA } \\
\hline \multicolumn{6}{|c|}{ Mức độ khó khăn vướng mắc của công tác QLCP hiện nay } \\
\hline & $\begin{array}{c}\text { Tổng các } \\
\text { bình phương }\end{array}$ & df & $\begin{array}{c}\text { Trung bình các } \\
\text { bình phương }\end{array}$ & $\mathrm{F}$ & Sig. \\
\hline $\begin{array}{c}\text { GIữa các } \\
\text { nhóm }\end{array}$ & 4,112 & 3 & 1,371 & 2,032 & 0,114 \\
\hline $\begin{array}{l}\text { Trong } \\
\text { các } \\
\text { nhóm }\end{array}$ & 68,136 & 101 & 0,675 & & \\
\hline Tổng & 72,248 & 104 & & & \\
\hline
\end{tabular}

Theo kết quả kiểm tra tính đồng nhất của các phương sai, với mức ý ngĩa Sig. = 0,113 > 0,05 (Bảng 13), có thể nói phương sai đánh giá mức độ khó khăn vướng mắc giữa các thâm niên không khác nhau một cách có ý nghĩa thống kê. Như vậy, kết quả phân tích ANOVA có thể sử dụng được.

Theo kết quả phân tích ANOVA, với mức ý ngĩa Sig. $=0,114>$ 0,05 (Bảng 2.14) nên có thể kết luận không có sự khác biệt có ý nghĩa thống kê về đánh giá mức độ khó khăn vướng mắc giữa các cá nhân tham gia khảo sát từ các thâm niên khác nhau.

\section{Kết luận và kiến nghị}

\subsection{Kết luân}

Bằng phương pháp tham vấn ý kiến chuyên gia. Các phiếu khảo sát được đưa vào chương trình SPSS V26. Dựa vào các kết quả kiểm định thang đo, phân tích nhân tố khám phá (EFA), phân tích hồi quy đa biến, dò tìm sự vi phạm giả định cần thiết trong hồi quy, phân tích phương sai một yếu tố (One_Way ANOVA). Kết quả đã xác định được các yếu tố ảnh hưởng đến mức độ khó khăn vướng mắc của công tác quản lý chi phí đầu tư xây dựng như sau:

Bảng 15.

Các yếu tố ảnh hưởng đến mức độ khó khăn vướng mắc trong công tác quản lý chi phí đầu tư xây dựng.

\begin{tabular}{|c|l|c|}
\hline STT & \multicolumn{1}{|c|}{ Yếu tố ảnh hưởng } & $\begin{array}{c}\text { Mức độ ảnh } \\
\text { hưởng }(\boldsymbol{\beta})\end{array}$ \\
\hline 1 & $\begin{array}{l}\text { Yếu tố văn bản pháp lý quản lý về chi phí đầu } \\
\text { tư xây dựng }\end{array}$ & 0,374 \\
\hline 2 & $\begin{array}{l}\text { Yếu tố năng lực, kinh nghiệm và ý thức trách } \\
\text { nhiệm của người tham gia quản lý chi phí đầu } \\
\text { tư xây dựng }\end{array}$ & 0,361 \\
\hline 3 & Yếu tố sự biến động giá cả thị trường & 0,301 \\
\hline 4 & $\begin{array}{l}\text { Yếu tố trang thiết bị, công nghệ, ứng dụng } \\
\text { trong công tác quản lý chi phí đầu tư xây dựng }\end{array}$ & 0,222 \\
\hline 5 & $\begin{array}{l}\text { Yếu tố quản lý công tác lập, thẩm tra, phê } \\
\text { duyệt thiết kế dự toán của chủ đầu tư }\end{array}$ & 0,220 \\
\hline
\end{tabular}

\subsection{Kiến nghị và hạn chế}

Căn cứ vào các yếu tố ảnh hưởng đã xác định ở bên trên, tác giả đưa ra một vài kiến nghị như sau:

Về phía nhà nước: Nhà nước cần ban hành những quy định, văn bản hướng dẫn về quản lý chi phí đầu tư xây dựng công trình một cách đồng bộ và thống nhất hơn. Tạo lập cơ sở dữ liệu điện tử, đồng bộ về định mức, đơn giá, giá vật liệu, lương nhân công, giá ca máy, chỉ số 
giá,...Có cơ chế chính sách cụ thể và khuyến khích ứng dụng khoa học công nghệ vào công tác quản lý chi phí đầu tư xây dựng nói riêng và toàn ngành xây dựng nói chung.

Về phía các chủ đầu tư: Tăng cường công tác đào tạo nhân lực, nâng cao ý thức trách nhiệm của người lao động. Có biện pháp mạnh với những cá nhân cố tình buông lỏng quản lý, thông đồng, hình thức, qua loa. Chú trọng đầu tư thiết bị công nghệ, khuyến khích các cá nhân tiên phong cải tiến, ứng dụng công nghệ số.

Kết quả của nghiên cứu này, chịu ảnh hưởng lớn bởi công tác thu thập và khảo sát dữ liệu. Chính vì thế vẫn còn mang tính chủ quan của tác giả, chưa phản ánh hết độ chính xác và toàn diện. Tuy nhên, nghiên cứu này cũng là cơ sở, nền tảng để thực hiện các nghiên cứu tiếp theo, nhằm tìm ra giải pháp sâu rộng, khắc phục các hạn chế khó khăn trong công tác quản lý chi phí đầu tư xây dựng vào thời gian tới.

\section{Tài liệu tham khảo}

[1].Hoàng T. and Chu Nguyễn Mộng N., Phân tích dữ liệu nghiên cứu với SPSS. NXB Hồng Đức, 2008.

[2].Nguyễn Đình T., Giáo trình phương pháp nghiên cứu khoa học trong kinh doanh. NXB Tài chính, 2013. 